\title{
Olfactory training assists in olfactory recovery after sinonasal
}

\section{surgery}

\author{
Joon Yong Park ${ }^{1}$, Jiyeon Lee ${ }^{2}$, Hansol Kim${ }^{1}$, Taesik Jung${ }^{1}$, and Jin Kook Kim ${ }^{1}$ \\ ${ }^{1}$ Konkuk University School of Medicine \\ ${ }^{2}$ Konkuk University Chungju Hospital
}

June 7,2021

\begin{abstract}
Abstract Objectives: In patients with chronic rhinosinusitis (CRS), olfactory dysfunction (OD) is a common complaint. The focus of this research was to evaluate the effectiveness of olfactory training (OT) in patients with CRS after sinonasal surgery. Design : Prospective controlled trial. Participants: We enrolled 111 Korean CRS patients who underwent sinonasal surgery. Main Outcome Measures: At baseline and three months after starting OT, both participants were assessed by an olfactory function test and endoscopy. The Korean version of the Sniffin' stick II (KVSS-II) was used to perform the olfactory function test. A visual analog scale and the Mini-Mental State Examination were used to assess nasal and psychological functions. Over the course of three months, five odorants were used in OT (rose, lemon, cinnamon, orange, and peach). Results: Over a 12-week duration, OT increased olfactory activity in nearly 62 percent of subjects than in non-OT subjects. The OT group had significantly higher olfactory outcomes for the total KVSS-II and identification scores than the non-OT group between the initial and follow-up assessments. The initial score influenced the degree of olfactory improvement after OT. Conclusions: OT patients exhibited significantly higher total KVSS-II scores compared with non-OT patients after sinonasal surgery; in particular, the odor identification score was different between the two groups. The results of this study show that a 12 -week period of repeated short-term exposure to various odors could be useful in enhancing olfactory activity in patients who underwent sinonasal surgery for the improvement of sensory-neural olfactory impairment.
\end{abstract}

\section{Abstract}

Objectives: In patients with chronic rhinosinusitis (CRS), olfactory dysfunction (OD) is a common complaint. The focus of this research was to evaluate the effectiveness of olfactory training (OT) in patients with CRS after sinonasal surgery.

Design : Prospective controlled trial.

Participants: We enrolled 111 Korean CRS patients who underwent sinonasal surgery.

Main Outcome Measures: At baseline and three months after starting OT, both participants were assessed by an olfactory function test and endoscopy. The Korean version of the Sniffin' stick II (KVSS-II) was used to perform the olfactory function test. A visual analog scale and the Mini-Mental State Examination were used to assess nasal and psychological functions. Over the course of three months, five odorants were used in OT (rose, lemon, cinnamon, orange, and peach).

Results: Over a 12-week duration, OT increased olfactory activity in nearly 62 percent of subjects than in non-OT subjects. The OT group had significantly higher olfactory outcomes for the total KVSS-II and identification scores than the non-OT group between the initial and follow-up assessments. The initial score influenced the degree of olfactory improvement after OT. 
Conclusions: OT patients exhibited significantly higher total KVSS-II scores compared with non-OT patients after sinonasal surgery; in particular, the odor identification score was different between the two groups. The results of this study show that a 12-week period of repeated short-term exposure to various odors could be useful in enhancing olfactory activity in patients who underwent sinonasal surgery for the improvement of sensory-neural olfactory impairment.

\section{Key points}

1. The aim of this study was to evaluate the effectiveness of olfactory training (OT) in patients with CRS after sinonasal surgery. 2. Patients were divided into an OT group that received frequent short-term exposure to various odors over a 12-week period, and their olfactory function was compared to that of a control group that did not receive post-operative OT. 3. OT patients exhibited significantly higher total TDI scores compared to non-OT patients at the end of 3 months. In the subset analysis, the odor identification scores differed between the two groups after 3 months. 4. Age, sex, median duration, and LM score at baseline assessment were not correlated with recovery of olfactory function. 5. The group with anosmia at baseline assessment showed a significantly higher increase in KVSS-II test results compared with the other patients

Keywords: Sinonasal surgery; Olfactory recovery; Olfactory training

\section{Introduction}

Olfactory dysfunction (OD) can be classified into conductive and sensorineural types, although these are not mutually exclusive. In conductive losses, such as nasal polyps and chronic rhinosinusitis (CRS), inspired odorants are unable to enter the olfactory cleft in the nasal cavity. In sensorineural loss, the damage of olfactory receptor neurons or their central projection contribute to OD. ${ }^{1}$ Attempted treatments have included medical (topical and systemic steroids, zinc, etc.) and surgical treatment. Hummel et al. ${ }^{2}$ studied the effectiveness of olfactory training $(\mathrm{OT})$ in a group of patients with olfactory loss due to post-infectious, posttraumatic, or idiopathic etiologies. OT has shown promise as an alternative treatment modality for several causes of OD, with the exception of sinonasal disease.

Previous research found that exposing various odors in patients with post-infectious and post-traumatic OD for 16 weeks increased their olfactory function. ${ }^{3}$ According to a recent meta-analysis, OT is a promising clinical therapy for patients with OD, and many other trials have shown that OT has good olfactory outcomes with no serious side effects. ${ }^{4}$ Recently, we reported that OT resulted in olfactory improvement reflected in the total threshold, discrimination and identification (TDI) score, threshold score, and identification score in patients with post-infectious olfactory dysfunction (PIOD) as compared to a control group using five odorants familiar to Koreans. ${ }^{5}$ Moreover, OT can be considered for patients with persistent COVID-19-related OD because this therapy is inexpensive and has negligible adverse effects. ${ }^{6}$

In patients with chronic rhinosinusitis (CRS), OD is a common complaint. ${ }^{7-9}$ Although the main mechanism of CRS-induced OD is unclear, it is thought to be a combination of mechanical obstruction from edematous mucosa or polyposis, as well as sensorineural damage from chronic inflammatory injury to the olfactory neuroepithelium. ${ }^{10}$ Endoscopic sinonasal surgery (ESS) is used to improve sinus function and access to topical medical treatment in patients with medically refractory CRS. However, olfactory function after ESS can be unpredictable. ${ }^{11-12}$ An early study endorsed the effect of ESS in reducing CRS-related OD. However, several recent studies on the outcomes of OD after ESS have reported conflicting results. ${ }^{13}$ A study found that OT improved the olfactory activity of sinonasal patients, but did not evaluate the effects of OT on post-sinonasaloperative patients. A separate study reported that no significant changes in individual component values of TDI scores were observed in sinonasal patients. However, that research did not include a control group. ${ }^{14}$ Therefore, the effectiveness of OT in CRS patients who experienced mixed (conductive and sensorineural) olfactory dysfunction after resolving the conductive cause by sinonasal surgery has not been investigated.

Objectives 
The aim of this study was to evaluate the effectiveness of olfactory training (OT) in patients with CRS after sinonasal surgery compared to non-OT patients.

\section{Methods}

\section{Ethical considerations}

This study was performed in accordance with the principles of the Declaration of Helsinki. The study was reviewed by the Institutional Review Board of [removed for blind peer review], and written informed consent was obtained from all patients. The study design was approved by the ethics committee of the medical faculty at the [removed for blind peer review].

\section{Participants}

Adult CRS patients with or without nasal polyps who required sinonasal surgery and were unresponsive to medication treatment were recruited from a sub-specialized rhinology clinic. To be included, the patients had to be [?] 18years of age with bilateral chronic or recurrent rhinosinusitis, and have a Lund-MacKay (LM) CT scan score difference [?] 3 between the right and left sides of the nasal cavity. Patients were excluded if they were unable or unwilling to comply with the required postoperative visits for data collection, or if they were ineligible for informed consent, displayed unilateral disease, had an olfactory cleft obstruction on preoperative CT scan or pre/perioperative endoscopic finding, had an underlying bleeding disorder, or a CT scan revealed a significant difference in disease status between the nasal cavities (LM score difference $>3$ ).

\section{Design}

Sinonasal surgery was performed to improve the conductive cause of OD. Surgery was performed by one surgeon under general anesthesia. Penicillin-based antibiotics were intravenously administrated for two days. Patients were treated endoscopically with debridement once a week as outpatients for the first month after discharge from the hospital. Oral antibiotics and intranasal corticosteroids were administrated for two weeks. Saline nasal irrigation was administrated once a day during postoperative care. Patients were treated once a month till the third month after the second month. Patients were assessed by an olfactory function test until the third month. The perioperative sinus endoscopy (POSE) scoring system was performed on all patients by a single experienced otorhinolaryngologist.

Following bilateral sinonasal surgery, patients started OT over a period of 12 weeks after their nasal packing was removed. The details of the OT procedure was explained to all patients who underwent bilateral ESS and they were offered the choice of continuing with the training program or waiting for a spontaneous recovery. The OT protocol and counselling on behavior and lifestyle modification was performed in accordance with our previously published method. ${ }^{5}$ Briefly, The patients were exposed to five different odorants twice a day: rose, lemon, cinnamon, orange, and peach. These odorants were chosen to represent odors that Koreans are familiar with. They were instructed to sniff the odorants for 10 seconds each morning and evening, with a 10-second break between each odorant. With the exception of OT, postoperative medical treatment did not differ between groups. Both groups were evaluated at 4, 8 and 12 weeks after the operation.

\section{Main outcome measures}

The Korean version of the Sniffin' stick II (KVSS-II) was used to perform the olfactory function test. A visual analog scale and the Korean version of the Mini-Mental State Examination (K-MMSE) were used to assess nasal and psychological functions. Three months after the baseline examination, all subjects were reviewed using endoscopy and the KVSS-II..

The baseline LM score, POSE score, visual analogue scale (VAS) for olfactory ability, and KVSS II score (threshold, discrimination, identification) were collected. The LM scoring system was used to compare preoperative status. Likewise, the POSE scoring system was used for the comparison of postoperative status. Olfactory function was evaluated using KVSS-II. The LM score was used to describe the CT findings. This score ranges from 0 (complete lucency of all sinuses) to 24 (complete opacity of all sinuses). ${ }^{15}$ 


\section{Statistical analyses}

SPSS version.21.0 (SPSS Inc., Chicago, IL, USA) was used for statistical analysis. The mean \pm standard deviation or percentage (\%) was used to present the demographic and clinical data. The t-test for independent samples was used to make comparisons between the training patients and controls. For categorical data, Pearson's chi-square test was applied. Paired t-test was used to compare olfactory function in each group at baseline and 12 weeks afterwards. Univariable linear regression analysis was performed to investigate the relationship between changes in TDI scores and age, sex, duration of disease, the severity of OD, LM score, and OT. Factors with a p-value of $<0.1$ were subjected to multivariable linear regression analysis. Multivariable linear regression analysis was performed to investigate the relationship between changes in TDI scores and sex, the severity of OD, and OT.

\section{Results}

\section{Participant demographics}

We enrolled 111 Korean participants (mean age: $45 \pm 15.4$ years; range: $18-74$ years). There were 68 patients in the OT group (mean age: 45.6 years; 41 males and 27 females) and 43 patients in the non-OT group (mean age: 44 years; 23 men and 20 women). Bilateral ESS was performed on all patients, and septoplasty was performed in some patients (total 26 patients: 17 patients in the OT group and 9 patients in the non-OT group) who had nasal septal deviation. After sinonasal surgery, there were no issues other than some minor postoperative bleeding in the hospital. There were no differences between the study groups at the baseline assessment in terms of the LM score, initial TDI scores, initial VAS score, or POSE score. Table 1 shows the descriptive statistics of the findings.

The effects of OT after sinonasal surgery.

There were no significant differences in TDI scores between the two groups at baseline, and there were no significant differences in odor threshold, discrimination, or identification subset tests.

Following sinonasal surgery, patients who underwent OT showed a significant increase in TDI scores after 3 months. The subset analysis of the TDI scores showed that the odor threshold, discrimination, and identification scores increased significantly in the OT group. The non-training group also showed improvement in the threshold score compared with the other scores, but the difference was not statistically significant. In either group, there were no significant variations in VAS scores between the test times. Table 2 shows the descriptive statistics for the outcomes.

OT patients exhibited significantly higher total TDI scores compared to non-OT patients at the end of 3 months. In the subset analysis, the odor identification scores differed between the two groups after 3 months. Table 1 and Figure 1 show the descriptive statistics of the findings.

Comparison of olfactory recovery by sex, age, severity, and duration of olfactory dysfunction

When the KVSS-II scores were adjusted for sex and degree of dysfunction, OT showed a significant effect on the difference in the KVSSII score $(\mathrm{p}<0.05)$. Age, median duration, and LM score at baseline assessment were not correlated with recovery of olfactory function in univariable linear regression analysis. Following adjustment for the degree of dysfunction and olfactory training factors, sex had no correlation with the difference in KVSS-II score. The group with anosmia at baseline assessment showed a significantly higher increase in KVSS-II test results compared with the other patients. Table 3 shows descriptive data for the outcomes.

\section{Discussion}

The following are the significant findings among CRS patients from this study: 1) When compared to non-OT patients, those who received OT showed statistically significant improvements in both total TDI score and identification score. 2) Differences in KVSS-II scores were independent of age, sex, LM score, and duration 
of disease and 3) Patients with anosmia at baseline assessment showed a significantly higher increase in the KVSS-II score compared with the other patients.

In this study, the patients who underwent sinonasal surgery with OT showed a significant improvement in TDI scores after 3 months. The subset analysis of the TDI scores showed that the odor threshold, discrimination, and identification scores increased significantly in the OT group. The non-training group also showed improvement in the threshold score compared with the other scores, but the difference was not statistically significant. According to a study of 20 trials published since 1991, olfaction generally improved following functional ESS. However, there has been limited research on the impact of OT on individuals with sinonasal disorders. ${ }^{16}$ There are also controversies about whether olfactory function improves with ESS in CRS patients. According to one prospective study, the University of Pennsylvania Smell Identification Test (UPSIT) score in the Chronic rhinosinusitis with nasal poloyp (CRSwNP) subgroup improved considerably following endoscopic sinus surgery. The evaluated UPSIT olfactory function improved after surgery in $46.0 \%$ of patients, did not change after surgery in $12.7 \%$ of patients, and worsened in $41.3 \%$ of patients. ${ }^{17}$ A 5 -year prospective research with $75 \%$ of the participants having CRSwNP found a considerable improvement in measured olfaction at 2 years after surgery, however this became non-significant at 5 years. ${ }^{18}$ There were also studies that reported negative olfactory outcomes after ESS. One study reported that the mean postoperative T\&T recognition threshold test of the eosinophilic CRS group declined after 12 months. ${ }^{19}$ From another study, $34 \%$ of patients had a decline in olfactory function after sinonasal surgery ${ }^{20}$ According to a meta-analysis of olfactory outcomes after ESS for CRS, ESS improved subjective and objective olfactory parameters, with patients with nasal polyposis and prior olfactory dysfunction showing the greatest benefits. ${ }^{21}$

We also observed significantly higher total TDI and identification scores in the OT group compared to nonOT patients. In our analysis, with the exception of the initial KVSS-II score, the difference in KVSSII score was independent of age, sex, LM score, and duration of disease. There were no significant differences between the OT and non-OT groups in terms of age, sex distribution, or duration of the condition in our prior study on PIOD patients. ${ }^{5}$ This is another significant difference compared to previous studies.

In this study of sinonasal patients, only the total TDI and identification score increased significantly. Our previous study on PIOD patients showed a significantly increased total TDI score, threshold score, and identification score after OT. The majority of prior research found that OT improved composite TDI, discrimination and identification scores in people with PIOD. The majority of prior research found that OT improved composite TDI, identification, and discrimination scores in people with PIOD. ${ }^{22}$

The odor threshold appears to be relatively unimpaired in central sources of olfactory dysfunction and is poorly connected with cognitive tests, therefore the threshold score is more strongly tied to peripheral abnormalities in the olfactory system. ${ }^{4}$ In this study, sinonasal surgery was performed in both groups and the surgical procedure improved the peripheral olfactory function and conduction of olfactory molecules. This could lead to improved threshold scores. Another KVSS-II score (total, identification) was significantly increased in the OT group, similar to our previous study on patients with PIOD.

The group with anosmia at baseline showed significantly higher improvements in KVSS-II score compared to the other patients. Long-term exposure to various odors enhances the survival of newly generated interneurons ${ }^{2}$ and odor memory, ${ }^{11}$ indicating that adult neurogenesis can play a role in olfactory memory. OT based on repeated stimulation by odors could promote the survival of immature new neurons and eliminate more mature neurons. ${ }^{23}$ Based on this mechanism, patients with lower olfactory function could improve significantly after OT.

There were some limitations to this study. First of all, only a small number of patients is involved. To better understand the effects of OT, larger studies are needed. Second, those who had less severe OD in the preoperative state could have been included in the non-OT group. This can be considered a selection bias. Third, OT was applied for only 12 weeks. Therefore, it is unclear if long-term OT exposure is helpful to olfactory function. 
In conclusion, OT patients exhibit significantly higher total TDI scores than non-OT patients after sinonasal surgery. In particular, odor identification scores were different between the two groups. According to the findings, a 12-week course of repeated short-term exposure to various odors could be useful in enhancing olfactory function in patients who had sinonasal surgery for sensory-neural OD.

\section{Reference}

[1] Goncalves S, Goldstein BJ. Pathophysiology of olfactory disorders and potential treatment strategies. Curr Otorhinolaryngol Rep 2016;4:115-121.

[2] Hummel T, Rissom K, Redden J, Hahner A, Weidenbecher M, Huttenbrink K-B. Effects of olfactory training in patients with olfactory loss. Laryngoscope 2009;119:496-499.

[3] Konstantinidis I, Tsakiropoulou E, Bekiaridou P, Kazantzidou C, Constantinidis J. Use of olfactory training in post-traumatic and postinfectious olfactory dysfunction. Laryngoscope 2013;123:E85-E90.

[4] Pekala K, Chandra RK, Turner JH. Efficacy of olfactory training in patients with olfactory loss: a systematic review and meta-analysis. Int Forum Allergy Rhinol 2016;6:299-307.

[5] Choi BY, Jeong H, Noh H, Park JY, Cho JH, Kim JK. Effects of olfactory training in patients with postinfectious olfactory dysfunction. Clin Exp Otorhinolaryngol 2021;14: 88-92.

[6] Whitcroft KL, Hummel T. Olfactory dysfunction in COVID-19: Diagnosis and management. JAMA 2020;323:2512-2514.

[7] Litvack JR, Mace JC, Smith TL. Olfactory function and disease severity in chronic rhinosinusitis. Am J Rhinol Allergy 2009;23:139-44.

[8] Soler ZM, Mace J, Smith TL. Symptom-based presentation of chronic rhinosinusitis and symptomspecific outcomes after endoscopic sinus surgery. Am J Rhinol 2008;22:297-301.

[9] Jiang RS, Lu FJ, Liang KL, Shiao JY, Su M-C, Hsin C-H. Olfactory function in patients with chronic rhinosinusitis before and after functional endoscopic sinus surgery. Am J Rhinol 2008;22:445-8.

[10] Raviv JR, Kern RC. Chronic sinusitis and olfactory dysfunction. Otolaryngologic Clin North Am 2004;37:1143-57.

[11] Yamagishi M, Hasegawa S, Suzuki S, Nakamura H, Nakano Y. Effect of surgical treatment of olfactory disturbance caused by localized ethmoiditis. Clin Otolaryngol Allied Sci 1989;14:405-409.

[12] Klimek L, Moll B, Amedee RG, Mann WJ. Olfactory function after microscopic endonasal surgery in patients with nasal polyps. Am J Rhinol 1997;11:251-255.

[13] Pade J, Hummel T. Olfactory function following nasal surgery. Larygoscope 2008;118:1260-1264.

[14] Fleiner F, Lau I, Göktas Ö. Active olfactory training for the treatment of smelling disorders. Ear, Nose Throat J 2012;91:198-215.

[15] Oluwole M, Russell N, Tan L, Gardiner Q, White P. A comparison of CT staging systems in chronic sinusitis. Clin Otolaryngol Allied Sci 1996;21:91-5.

[16] Hummel T, Whitcroft KL, Altundag A, Cinghi C, Costanzo RM, Damm M, et al. Position paper on olfactory dysfunction. Rhinol Suppl 2017;54:1-30.

[17] Andrews PJ, Poirrier A-L, Lund VJ, Choi D. Outcomes in endoscopic sinus surgery: olfaction, nose scale and quality of life in a prospective cohort study. Clin. Otolaryngol. 2016;41:798-803.

[18] Rowe-Jones JM, Medcalf M, Durham SR, Richards DH, Mackay IS. Functional endoscopic sinus surgery: 5 year follow up and results of a prospective, randomised, stratified, double-blind, placebo controlled study of postoperative fluticasone propionate aqueous nasal spray. Rhinology 2005;43:2-10. 
[19] Oka H, Tsuzuki K, Takebayashi H, Kojima Y, Daimon T, Sakagami M. Olfactory changes after endoscopic sinus surgery in patients with chronic rhinosinusitis. Auris Nasus Larynx 2013;40:452-457.

[20] Kimmelman CP. The risk to olfaction from nasal surgery. Laryngoscope 1994;104:981-988.

[21] Kohli P, Naik AN, Farhood Z, Ong AA, Nguyen SA, Soler ZM, et al. Olfactory outcomes after endoscopic sinus surgery for chronic rhinosinusitis: A meta-analysis. Otolaryngol Head Neck Surg 2016;155:936-948.

[22] Sorokowska A, Drechsler E, Karwowski M, Hummel T. Effects of olfactory training: a meta-analysis. Rhinology.2017;55:17-26.

[23] Mouret A, Gheusi G, Gabellec M-M, de Chaumont F, Olivo-Marin J-C, Lledo P-M. Learning and Survival of Newly Generated Neurons: When time matters. J Neurosci;2008;28:11511-11516.

Figure legends

Figure 1. In the olfactory training (OT) and non-OT groups, the total threshold, discrimination, and identification (TDI) score (A), threshold score (B), discrimination score (C), identification score (D), and difference between the initial and final evaluations are compared. KVSS-II, Korean version of Sniffin' stick. ${ }^{*} \mathrm{P}<0.05$

\section{Hosted file}

3. Table_1.docx available at https://authorea.com/users/418338/articles/525111-olfactorytraining-assists-in-olfactory-recovery-after-sinonasal-surgery

\section{Hosted file}

4. Table_2.docx available at https://authorea.com/users/418338/articles/525111-olfactorytraining-assists-in-olfactory-recovery-after-sinonasal-surgery

\section{Hosted file}

5. Table_3.docx available at https://authorea.com/users/418338/articles/525111-olfactorytraining-assists-in-olfactory-recovery-after-sinonasal-surgery 
(A)

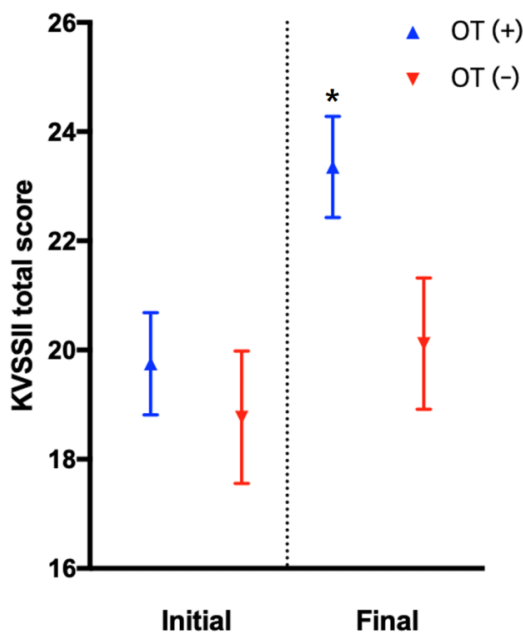

(C)

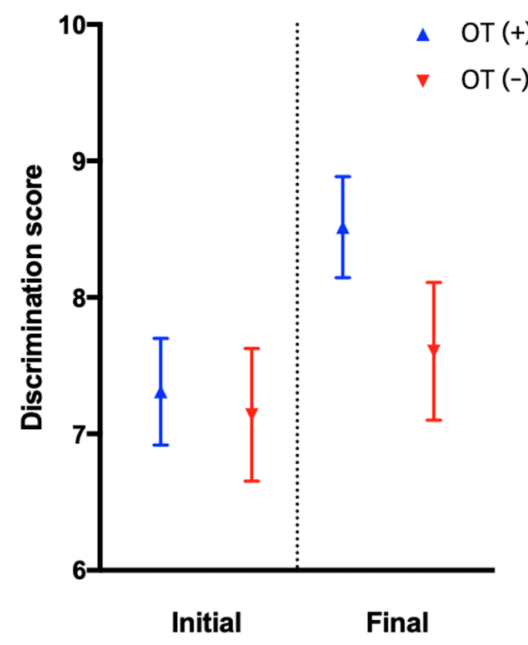

(B)

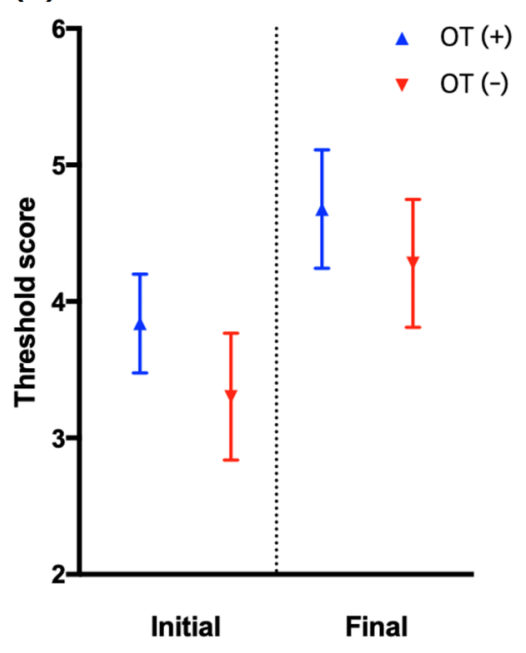

(D)

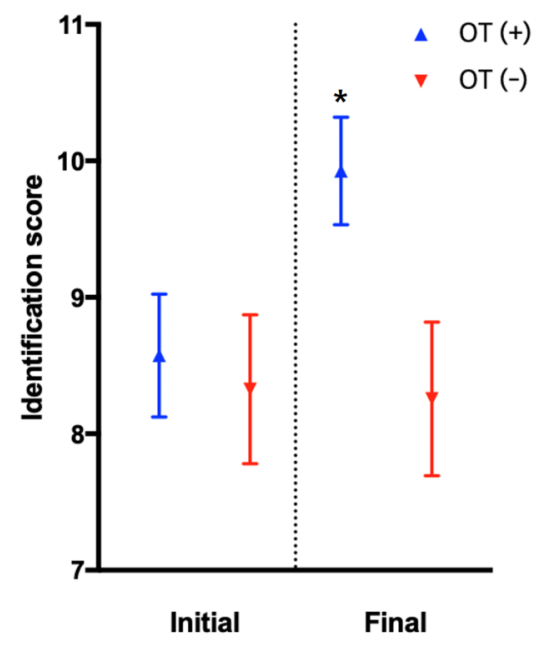

